\title{
Immunoglobulin G4-related Disease Accompanied by Peripheral Neuropathy: A Report of Two Cases
}

\author{
Tomoya Kawazoe ${ }^{1}$, Tomoyuki Inoue ${ }^{1}$, Shinsuke Tobisawa ${ }^{1}$, Keizo Sugaya ${ }^{1}$, Toshio Shimizu ${ }^{1}$, \\ Kazuhito Miyamoto ${ }^{1}$, Manaka Goto ${ }^{2}$, Naoto Yokogawa ${ }^{2}$, Sanami Azuma $^{3}$, Shingo Itagaki ${ }^{4}$, \\ Kenji Nishida ${ }^{5}$, Yasuhiro Morita ${ }^{6}$, Masahiro Nagao ${ }^{1}$ and Eiji Isozaki ${ }^{1}$
}

\begin{abstract}
:
Due to its rarity and the limited literature, the clinicopathological characteristics of peripheral nerve involvement in immunoglobulin G4 (IgG4)-related disease are unknown. We present two cases of IgG4-related disease, accompanied by peripheral neuropathy, presenting as unilateral ptosis (case 1) and sclerosing cholangitis (case 2), respectively. In both cases, sural nerve biopsy indicated vasculitis as the underlying pathophysiology; the peripheral neuropathy was refractory to corticosteroid therapy. In contrast to the previously proposed pathomechanism of IgG4-related neuropathy (direct lymphoplasmacytic infiltration), the pathological findings in our cases suggest that vasculitis occurs secondary to systemic autoimmune conditions.
\end{abstract}

Key words: case report, IgG4-related disease, systemic autoimmune disease, peripheral neuropathy, vasculitic neuropathy

(Intern Med 60: 1941-1947, 2021)

(DOI: 10.2169/internalmedicine.6461-20)

\section{Introduction}

Immunoglobulin G4 (IgG4)-related disease is a relatively new and emerging systemic autoimmune condition characterized by a distinctive histopathological appearance of lymphoplasmacytic infiltrates with abundant IgG4-positive plasma cells, storiform fibrosis, and obliterative phlebitis (1). The first case of apparent IgG4-related disease was reported by Jan Mikulicz-Radecki, in the late 19th century, as a case of lacrimal gland swelling followed by submandibular and parotid gland swelling (2). In the late 20th century, Kawaguchi et al. reported two cases in patients undergoing surgery for pancreatic carcinoma; the pathological findings were lymphoplasmacytic sclerosing pancreatitis with cholangitis (3). Hamano et al. reported serum IgG4 elevation in patients with sclerosing pancreatitis (4). In 2012, IgG4related disease was described as a novel clinical entity (1).
Among the neurological involvements of IgG4-related disease, peripheral neuropathy is reported to be rare, occurring in $<1 \%$ of patients with this disease (5). In 2013, Ohyama et al. reported the first case of IgG4-related peripheral neuropathy with direct lymphoplasmacytic infiltration in a patient with local IgG4-related skin lesions (6). However, no further cases of peripheral neuropathy caused by direct cellular infiltration have been reported since then. Thus, the clinicopathological characteristics of peripheral neuropathy in IgG4related disease are unclear. We herein report two cases of IgG4-related disease with peripheral neuropathy.

\section{Case Reports}

\section{Case 1}

An 81-year-old Japanese woman presented with a 6month history of unilateral ptosis that appeared after a few

${ }^{1}$ Department of Neurology, Tokyo Metropolitan Neurological Hospital, Japan, ${ }^{2}$ Department of Rheumatic Diseases, Tokyo Metropolitan Tama Medical Center, Japan, ${ }^{3}$ Department of Otolaryngology-Head and Neck Surgery, Tokyo Metropolitan Tama Medical Center, Japan, ${ }^{4}$ Department of Pathology, Tokyo Metropolitan Tama Medical Center, Japan, ${ }^{5}$ Department of Endocrinology and Metabolism, Tokyo Metropolitan Tama Medical Center, Japan and ${ }^{6}$ Department of Surgery, Tokyo Metropolitan Tama Medical Center, Japan

Received: October 6, 2020; Accepted: November 27, 2020; Advance Publication by J-STAGE: January 15, 2021

Correspondence to Dr. Tomoya Kawazoe, tomoya_kawazoe@tmhp.jp 
Table 1. Nerve Conduction Study.

\begin{tabular}{|c|c|c|c|c|c|c|c|}
\hline \multirow[t]{2}{*}{ Age/sex } & & \multicolumn{2}{|c|}{$\begin{array}{c}\text { Case } 1 \\
81 / \mathrm{F}\end{array}$} & \multicolumn{2}{|c|}{$\begin{array}{c}\text { Case } 2 \\
69 / \mathrm{M}\end{array}$} & \multicolumn{2}{|c|}{ Controls } \\
\hline & & $\mathrm{L}$ & $\mathrm{R}$ & $\mathrm{L}$ & $\mathrm{R}$ & Mean & SD \\
\hline \multicolumn{8}{|l|}{ Median } \\
\hline $\mathrm{MCV}$ & $(\mathrm{m} / \mathrm{s})$ & 54 & 58 & 54 & 47 & 57.7 & 4.9 \\
\hline DL & (ms) & 3.4 & 3.1 & 3.6 & 3.1 & 3.49 & 0.34 \\
\hline CMAP & $(\mathrm{mV})$ & 7.1 & 6.0 & 9.7 & 9.0 & 7.0 & 3.0 \\
\hline SCV & $(\mathrm{m} / \mathrm{s})$ & 58 & 52 & 51 & 56 & 56.2 & 5.8 \\
\hline SNAP & $(\mu \mathrm{V})$ & 15.9 & 10.2 & 8.9 & 9.8 & 38.5 & 15.6 \\
\hline \multicolumn{8}{|l|}{ Ulnar } \\
\hline $\mathrm{MCV}$ & $(\mathrm{m} / \mathrm{s})$ & 56 & 61 & 51 & 48 & 58.7 & 5.1 \\
\hline DL & $(\mathrm{ms})$ & 2.4 & 2.7 & 2.6 & 2.3 & 2.59 & 0.39 \\
\hline CMAP & $(\mathrm{mV})$ & 11.4 & 11.4 & 9.1 & 7.9 & 5.7 & 2.0 \\
\hline SCV & $(\mathrm{m} / \mathrm{s})$ & 46 & 48 & 49 & 50 & 54.8 & 5.3 \\
\hline SNAP & $(\mu \mathrm{V})$ & 12.3 & 12.8 & 9.3 & 6.4 & 35.0 & 14.7 \\
\hline \multicolumn{8}{|l|}{ Tibial } \\
\hline $\mathrm{MCV}$ & $(\mathrm{m} / \mathrm{s})$ & 51 & 44 & 39 & 43 & 48.5 & 3.6 \\
\hline DL & (ms) & 4.0 & 3.7 & 3.4 & 3.5 & 3.96 & 1.00 \\
\hline CMAP & $(\mathrm{mV})$ & 8.2 & 11.4 & 8.6 & 12.9 & 5.8 & 1.9 \\
\hline \multicolumn{8}{|l|}{ Sural } \\
\hline SCV & $(\mathrm{m} / \mathrm{s})$ & 45 & $\mathrm{NE}$ & $\mathrm{NE}$ & $\mathrm{NE}$ & 51.1 & 5.9 \\
\hline SNAP & $(\mu \mathrm{V})$ & 3.1 & $\mathrm{NE}$ & $\mathrm{NE}$ & $\mathrm{NE}$ & 17.2 & 6.7 \\
\hline
\end{tabular}

The control values are based on the textbook by Jun Kimura, Oxford University Press, Electrodiagnosis in diseases of nerve and muscle: principles and practice, Third edition.

CMAP: compound muscle action potential, DL: distal latency, F: female, L: left, M: male, MCV: motor conduction velocity, ND: not described, NE: not elicited, R: right, SCV: sensory conduction velocity, SD: standard deviation, SNAP: sensory nerve action potential

days of eyelid swelling, and a 1-month history of plantar dysesthesia. Surprisingly, she had a longstanding medical history of immunological complications, starting with a 20year history of sinusitis and hypothyroidism due to thyroiditis. She developed sialadenitis at 66 years of age, allergic rhinitis at 67 years of age, and right submandibular gland enlargement at 68 years of age. However, from the 61 to 81 years of age, she did not receive any immunotherapy because her symptoms were thought to be non-specific or related to aging. A neurological examination at 81 years of age revealed left ptosis. With the exception of left ptosis (both pupils were equally reactive to light), and right predominant plantar dysesthesia, which she described as a sensation of bubbling water on her skin, she showed no further signs of neurological disturbance.

A laboratory examination revealed an elevated erythrocyte sedimentation rate $(79 \mathrm{~mm} / \mathrm{h}$, reference: $<17 \mathrm{~mm} / \mathrm{h})$ and hypergammaglobulinemia with beta-gamma $(\beta-\gamma)$ bridging without a monoclonal band. The serum levels of immunoglobulin $\mathrm{G}(\mathrm{IgG})(2,754 \mathrm{mg} / \mathrm{dL}$, reference: $<1,700 \mathrm{mg} / \mathrm{dL})$, IgG4 (1,310 mg/dL, reference: $<117 \mathrm{mg} / \mathrm{dL})$, and immunoglobulin E $(764 \mathrm{U} / \mathrm{mL}$, reference: $<170 \mathrm{U} / \mathrm{mL})$ were elevated. The anti-Ro/SSA antibody titer was $93 \mathrm{U} / \mathrm{mL}$ (reference: $<7 \mathrm{U} / \mathrm{mL}$ ) and the patient tested negative for anti-La/ SSB antibodies. The level of anti-thyroglobulin was 1,697
$\mathrm{U} / \mathrm{mL}$ (reference: $<28 \mathrm{U} / \mathrm{mL}$ ) and antithyroid peroxidase was $390 \mathrm{U} / \mathrm{mL}$ (reference: $<16 \mathrm{U} / \mathrm{mL}$ ). The patient tested negative for anti-neutrophil cytoplasmic and antiacetylcholine receptor antibodies. A nerve conduction study revealed an asymmetrical response in the right sural nerve (Table 1). Repetitive nerve stimulation showed normal neuromuscular transmission. Computed tomography (CT) showed retroperitoneal fibrosis, mediastinal and hilar lymphadenopathy, and enlargement of the thyroid gland and pancreas. Magnetic resonance imaging (MRI) revealed bilateral lacrimal gland swelling and enlargement of the left levator palpebrae superioris, indicating myositis or edema of the muscle (Fig. 1A; this improved after treatment, as shown in Fig. 1B).

Since the patient's concerns were plantar dysesthesia as well as ptosis, she requested a nerve biopsy. The sural nerve biopsy revealed epineural perivascular lymphocytic infiltration (Fig. 1C), vascular occlusion, loss of myelinated fibers, and myelin ovoids (Fig. 1D). To exclude malignancies or similar diseases (e.g., cancer, lymphoma, Sjögren's syndrome, multicentric Castleman disease, granulomatosis with polyangiitis, and eosinophilic granulomatosis with polyangiitis), we performed lip, submandibular lymph node, and nasal mucosal biopsies. The lip (Fig. 1E-H) and submandibular lymph node biopsies revealed lymphoplasmacytic infiltration with an IgG4-/IgG-positive cell ratio of $>70 \%$. Finally, a diagnosis of IgG4-related disease with enlargement of the levator palpebrae superioris and peripheral neuropathy was made.

Considering the patient's age, we treated her with oral prednisolone (10 mg/day; body weight, $45 \mathrm{~kg})$. After one month of treatment, the ptosis and salivation, which she had thought to be a sign of aging, improved. The treatment response indicated that the dry mouth was due to IgG4-related sialadenitis. The plantar dysesthesia remained unchanged. Improvement of the levator palpebrae superioris and lacrimal enlargement were confirmed on MRI (Fig. 1B). Improvement of the pancreatic enlargement, lymphadenopathy, and retroperitoneal fibrosis were confirmed on CT. The dose of prednisolone had to be reduced to $5 \mathrm{mg} /$ day due to the patient experiencing general fatigue, which improved without a relapse of other symptoms.

\section{Case 2}

A 69-year-old Japanese man was referred to us with limb numbness that had appeared one month before admission. At 68 years of age, he had undergone surgery for oral cancer (squamous cell carcinoma), which did not require chemotherapy or radiotherapy. At one year after surgery, followup CT showed right hilar and left axillary lymphadenopathy and a low-density area in the liver with a dilated left bile duct. Positron emission tomography/CT showed lesions in the liver and bilateral supraclavicular, right hilar, left axillary, and hepatoduodenal mesenteric lymph nodes. Biopsy of the right hilar and left axillary lymph nodes detected no evidence of malignancy. After the preoperative diagnosis of hi- 

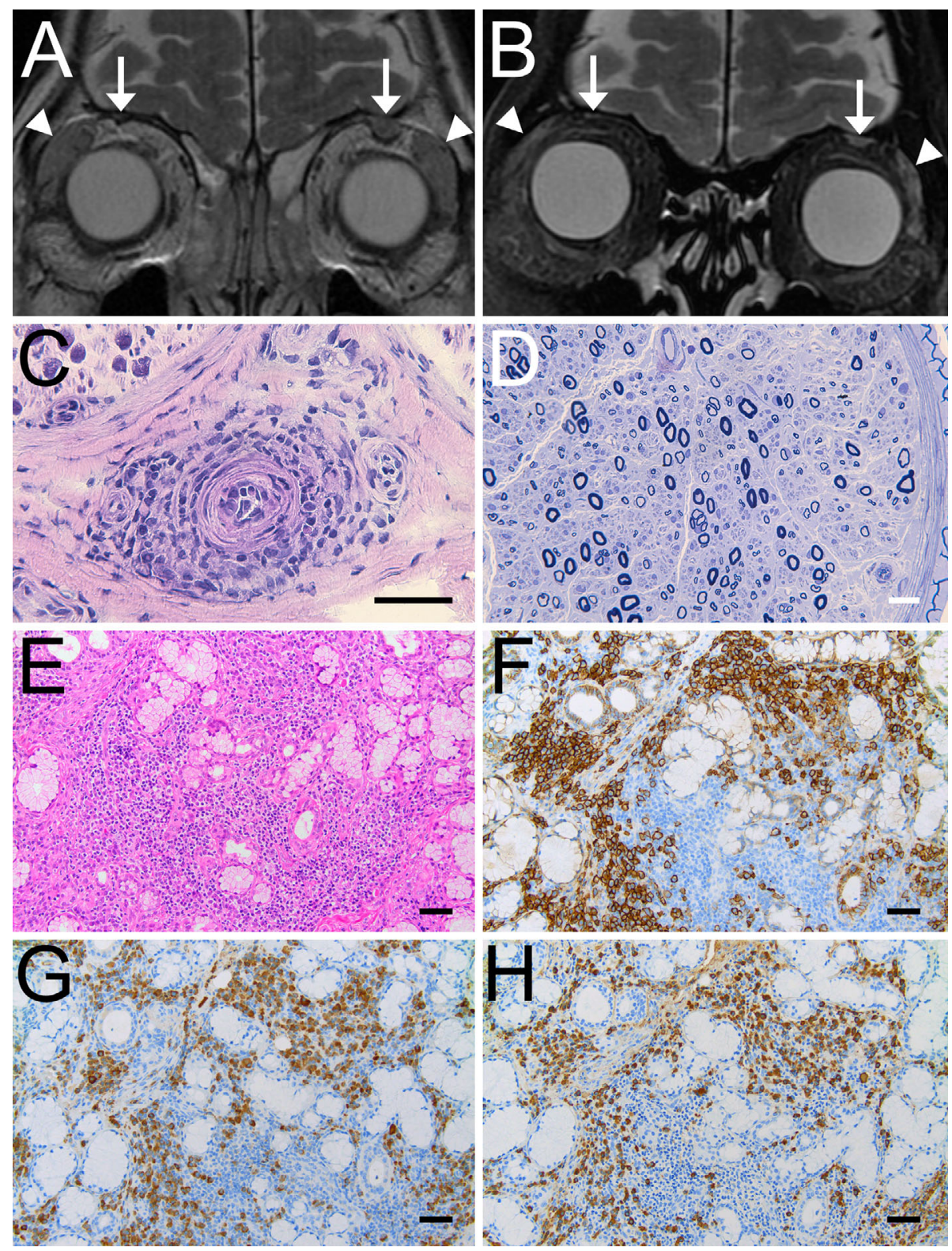

Figure 1. Radiological and pathological findings of the patient (case 1). A coronal T2-weighted image (A) and T2 fat-suppression image (B). (A) The initial presentation 81 years of age. The arrow indicates the levator palpebrae superioris muscle. The arrowhead indicates the lacrimal gland. Enlargement of the left levator palpebrae superioris and bilateral lacrimal glands is evident. (B) After treatment with prednisolone $(10 \mathrm{mg} /$ day $)$ for one month, the lacrimal glands returned to a normal size and the enlargement of the levator palpebrae superioris in the left eye was reduced. Sural nerve biopsy (C, D). (C) Hematoxylin and Eosin (H\&E) staining. Epineural perivascular lymphocytic infiltration was evident. IgG4-positive cells were not evident (not shown). (D) Epoxy resin-embedded transverse sections stained with toluidine blue. Reduction of the myelinated fibers, and thin myelinated clusters were observed, indicating the occurrence of axonal degeneration. Lip biopsy (E-H). (E) H\&E staining. Marked inflammatory cell infiltration was observed. Duct destruction was not evident. (FH) Immunohistochemistry. (F) Infiltration of CD138-positive plasma cells, which were stained in brown, was evident. (G, H) IgG4-positive cells $(\mathrm{H})$ accounted for $>70 \%$ of the IgG-positive cells (G). Scale bars indicate $50 \mu \mathrm{m}$.

lar cholangiocarcinoma (cT2bN1M0, stage IIIb), left hepatectomy, caudal lobectomy, extrahepatic bile duct resection, and choledochojejunostomy were performed. The resected specimen included a well-circumscribed mass lesion (Fig. 2A). Unexpectedly, no malignancy was identified; in- stead, storiform fibrosis (Fig. 2B) and lymphoplasmacytic infiltration (Fig. 2C) with IgG4-positive cells (Fig. 2D) were observed.

At two months after surgery, the patient developed limb numbness (bilateral planter dysesthesia appeared, which as- 

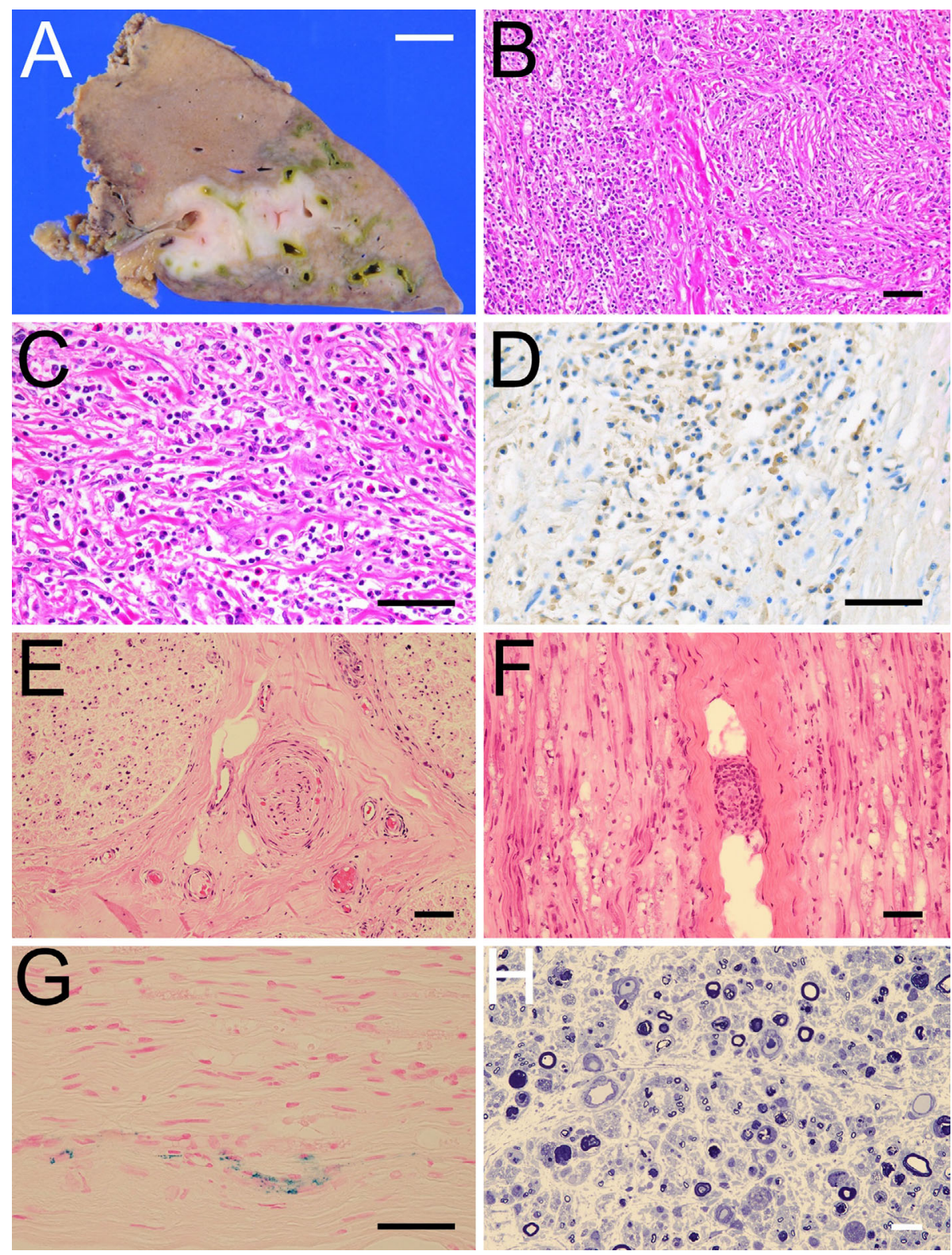

Figure 2. Surgical and pathological findings of the patient (case 2). (A-D) Sclerosing cholangitis. (A) The resected left liver and caudate lobe $(19 \mathrm{~cm} \times 10 \mathrm{~cm} \times 5 \mathrm{~cm})$, including a pseudotumor-like lesion $(3.2$ $\mathrm{cm} \times 3.0 \mathrm{~cm} \times 1.6 \mathrm{~cm})$ at the hepatic hilum. (B) Hematoxylin and Eosin (H\&E) staining showed storiform fibrosis and obliterative phlebitis. (C) H\&E staining. (D) Immunohistochemistry. Lymphoplasmacytic infiltration (C) with IgG4-positive cells (D) was evident, accounting for $>\mathbf{4 5 \%}$ of the IgGpositive cells, with more than 50-60 IgG4-positive plasma cells per high-power field. (E-H) Sural nerve biopsy. (E-G) H\&E staining. Vascular occlusion, vascular recanalization, and epineural fibrosis were observed (E). Epineural lymphocytic infiltration was observed (F) without evident IgG4-positive cells (not shown). Hemosiderin deposits are shown (G). (H) Epoxy resin-embedded transverse sections stained with toluidine blue. Myelin ovoids were evident. Scale bars indicate $1 \mathrm{~cm}$ in $\mathrm{A}$ and $50 \mu \mathrm{m}$ in $\mathbf{B}-\mathrm{H}$.

cended to the knee within a week and was soon followed by dysesthesia of both hands). A neurological examination revealed bilateral weakness of the distal lower limbs (grade 4 according to the Medical Research Council Scale for Muscle Strength), sensory disturbance (decreased sensations of touch and vibrations in the distal lower limbs), and abnormal ankle jerk (absent on the left side and decreased on the right side).
A laboratory examination revealed hypergammaglobulinemia with $\beta-\gamma$ bridging without a monoclonal band. The serum $\mathrm{IgG}$ level was $1,865 \mathrm{mg} / \mathrm{dL}$ (reference: $<1,700 \mathrm{mg} / \mathrm{dL}$ ) and the IgG4 level was elevated at $712 \mathrm{mg} / \mathrm{dL}$ (reference: < $117 \mathrm{mg} / \mathrm{dL}$ ). Autoantibody screening was negative. A nerve conduction study found axonal neuropathy with a pattern of mononeuritis multiplex (Table 1). Sural nerve biopsy detected vascular occlusion, recanalization, and epineural fi- 
brosis (Fig. 2E). Moreover, lymphocytic infiltration without IgG4-positive cells (Fig. 2F), hemosiderin deposition (Fig. 2G), and axonal neuropathy (Fig. 2H) were observed. Based on these results, a diagnosis of mononeuritis multiplex complicated with IgG4-related sclerosing cholangitis was made. The patient was treated with oral prednisolone $(0.5 \mathrm{mg} / \mathrm{kg} / \mathrm{day})$, and after one month his muscle weakness fully recovered; however, the sensory disturbances remained unchanged.

\section{Discussion}

In case 1 , the patient's longstanding immunological history of sinusitis, thyroiditis (Riedel's), sialadenitis (Mikulicz's disease, Küttner's tumor), rhinitis, orbital disease, peripheral neuropathy, retroperitoneal fibrosis (Ormond's disease), pancreatitis, and lymphadenopathy were explained by a single etiology of IgG4-related disease as the underlying cause. Regarding sclerosing cholangitis and pancreatitis, due to the non-specific signs and pseudotumor-like lesions, patients with IgG4-related disease are often misdiagnosed with a malignancy. In some cases, however, IgG4related pathophysiology is identified on the biopsy before the surgical procedure (7). As in case 2, when the patient is asymptomatic and in the absence of specific biopsy findings, surgery is inevitably required to establish a diagnosis. In retrospect, the laboratory findings in case 2, of hypergammaglobulinemia with $\beta-\gamma$ bridging, which were highlighted as a key feature in the report by Hamano et al. (4), was a clue to diagnosis prior to the surgical intervention.

In clinical practice, our cases highlight two noteworthy points. First, regarding the ptosis in case 1, although ophthalmic involvement is frequent in IgG4-related disease, leading to IgG4-related ophthalmic disease (8), IgG4-related disease is often neglected in the differential diagnosis of ptosis. To our knowledge, there is no report on ptosis caused by the enlargement of the levator palpebrae superioris due to IgG4-related disease. In case 1, unilateral enlargement of the levator palpebrae superioris muscle was revealed by MRI, and myositis or edema was noticed. Among the differential diagnoses of orbital myositis associated with systemic inflammatory and autoimmune disease (except for thyroidassociated orbitopathy), IgG4-related disease is the most frequently noted, with a prevalence of $15-37 \%$ among patients with IgG4-related disease; however, extraocular muscle involvement mainly focuses on muscles other than the levator palpebrae superioris (9). In case 1, ptosis was the initial clue to the diagnosis; hence, enlargement of the levator palpebrae superioris due to IgG4-related disease should be considered in the differential diagnosis of patients with ptosis.

Second, although peripheral neuropathy is known to be complicated with IgG4-related disease, with scarce case reports, little is known about its clinicopathological characteristics (5). The neurological manifestations of IgG4-related disease are common in hypertrophic pachymeningitis, hypophysitis, inflammatory pseudotumors, and parenchymal brain involvement, while neuropathy is rare (except in the orbital or paravertebral areas), with a frequency of $<1 \%$ among patients with IgG4-related disease (10). Until now, most reports on IgG4-related neuropathy have addressed the focal involvement of the nerve adjacent to the IgG4-related perineural lesion, particularly in the orbital area, and reports on peripheral neuropathy of the limbs are scarce (11).

Regarding the pathological diagnosis of neuropathy, without evident IgG4-positive plasmacytic infiltration, neither of the cases fulfilled the histological criteria for IgG4-related disease; instead, both cases met the diagnostic histopathological criteria for probable vasculitic neuropathy (Peripheral Nerve Society Guideline 2010), with findings of primary axonal neuropathic changes without significant demyelination, perivascular inflammation, or chronic vascular damage (12). Table 2 presents a summary of cases of IgG4related disease complicated by peripheral neuropathy. Ohyama et al. analyzed 149 consecutive patients with inflammatory neuropathies and reported that $\mathrm{IgG} 4$ was involved, especially in patients with primary systemic vasculitis (13). We propose two different pathomechanisms underlying peripheral neuropathy related to IgG4-related disease. One is consistent with the characteristic pathological findings of IgG4-related disease, and the other is associated with vasculitis due to a systemic autoimmune condition without significant infiltration of IgG4-positive plasma cells.

In 2013, Ohyama et al. reported the first case of IgG4related peripheral neuropathy with marked fibrosis and epineural IgG4-positive plasmocytic infiltration (6). The patient showed local skin lesions in the lower limbs without systemic involvement of IgG4-related disease. Since this report, no case of IgG4-related neuropathy with a similar pathophysiology has been described, suggesting that focal perineural infiltration adjacent to the skin lesion occurred in the first case by Ohyama et al. The same group reported a case of neuropathy with tumefactive cellular infiltration without IgG4-positive plasma cells or fibrosis in a patient with generalized IgG4-related lymphadenopathy (14). Additionally, Suzuki et al. described the case of a patient with anemia and limb dysesthesia with pain who underwent a bone marrow biopsy with a clinical diagnosis of multiple myeloma. The patient unexpectedly showed marked IgG4positive lymphoplasmacytic infiltration in the bone marrow, leading to the diagnosis of IgG4-related disease, and excluding multiple myeloma (15).

From the viewpoint of therapy, in the case reported by Ohyama et al., the patient responded well to prednisolone (6). Importantly, in the case reported by Suzuki et al., the symptoms of neuropathy were refractory to prednisolone (15). Contrarily, other IgG4-related symptoms, including those of the bone marrow, were resolved with prednisolone treatment. In our cases, the response to prednisolone was refractory (Case 1) or partial (Case 2) (Table 2), although it is possible that Case 1 could have responded if we had administered a higher dose of prednisolone, such as 30 $\mathrm{mg} /$ day [the recommended glucocorticoid regimen for the 
Table 2. Summary of the Patients with IgG4-related Disease, with Systemic or Local Involvement, Accompanied by Peripheral Neuropathy.

\begin{tabular}{|c|c|c|c|c|c|}
\hline & Case 1 & Case 2 & $\begin{array}{c}\text { Reference } \\
\text { (14) }\end{array}$ & $\begin{array}{c}\text { Reference } \\
\text { (15) }\end{array}$ & $\begin{array}{l}\text { Reference } \\
\text { (6) }\end{array}$ \\
\hline Age/sex & $81 / \mathrm{F}$ & $69 / \mathrm{M}$ & $56 / \mathrm{M}$ & $78 / \mathrm{M}$ & $55 / \mathrm{M}$ \\
\hline Serum IgG4 (mg/dL) & 1,310 & 712 & 328 & 1,290 & 259 \\
\hline Serum IgG4/IgG (\%) & 47 & 38 & 10 & 24 & 10 \\
\hline Systemic involvements & $\begin{array}{c}\text { PNS } \\
\text { Thyroid } \\
\text { SG SMG } \\
\text { LG } \\
\text { EOM } \\
\text { RPF } \\
\text { Pancreas } \\
\text { Ly }\end{array}$ & $\begin{array}{l}\text { SC } \\
\text { Ly }\end{array}$ & $\begin{array}{c}\text { Ly } \\
\text { HSM }\end{array}$ & $\begin{array}{c}\text { BM } \\
\text { HSM } \\
\text { Ly }\end{array}$ & $\begin{array}{c}\text { None } \\
\text { (Local skin } \\
\text { lesion) }\end{array}$ \\
\hline IgG4-pathology-confirmed tissues & $\begin{array}{l}\text { Lip } \\
\text { SMG }\end{array}$ & Bile duct & $\begin{array}{l}\text { Lymph } \\
\text { node }\end{array}$ & $\mathrm{BM}$ & Skin \\
\hline \multicolumn{6}{|l|}{ Sural nerve biopsy } \\
\hline IgG4-positive lymphoplasmacytic invasions & - & - & - & - & + \\
\hline Suggestive of vasculitis & + & + & + & + & - \\
\hline Type of IgG4-RD & Systemic & Systemic & Systemic & Systemic & Local \\
\hline IVMP & - & - & - & + & - \\
\hline
\end{tabular}

ANA: antinuclear antibody, BM: bone marrow, DNA: anti-DNA antibody, EOM: extraocular muscle, HSM: hepatosplenomegaly, IgG4RD: IgG4-related disease, IVMP: intravenous methylprednisolone, LG: lacrimal grand, Ly: lymphadenopathy, ND: not described, NT: not tested, PNS: paranasal sinus, RPF: retroperitoneal fibrosis, SC: sclerosing cholangitis, SG: salivary gland, SMG: submandibular gland, Ro/ SSA: anti-Ro/SSA antibody, La/SSB: anti-La/SSB antibody, Tg: anti-thyroglobulin antibody, TPO: anti-thyroid peroxidase antibody

treatment of IgG4-related disease is prednisolone or prednisone at a dose of $0.4-0.6 \mathrm{mg} / \mathrm{kg} /$ day (16)]. Contrary to the case reported by Ohyama et al. (6) in which the condition was limited to a local response, our cases, together with the cases reported by Yokoi et al. and Suzuki et al. $(14,15)$, imply that systemic autoimmune conditions are involved in the pathogenesis of neuropathy related to IgG4-related disease (Table 2).

To conclude, we presented two cases of IgG4-related disease with ptosis (case 1) and a pseudotumor of the bile duct (case 2). These cases have educational value and contribute to medical knowledge by highlighting the need to include IgG4-related disease in the differential diagnosis of ptosis and cholangiocarcinoma, and by emphasizing the importance of the laboratory finding of hypergammaglobulinemia with $\beta-\gamma$ bridging as a clue to the diagnosis. Furthermore, in addition to the previously proposed IgG4-related peripheral neuropathy as focal invasion, our cases suggest that a type of vasculitis occurs secondary to the systemic IgG4-related conditions.
Written informed consent for publication was obtained from the patients.

The authors state that they have no Conflict of Interest (COI).

\section{References}

1. Umehara H, Okazaki K, Masaki Y, et al. A novel clinical entity, IgG4-related disease (IgG4RD): general concept and details. Mod Rheumatol 22: 1-14, 2012.

2. Mikulicz J. About a peculiar symmetrical disease of the lacrimal and salivary glands. Beitrage zur Chirurgie-Festschrift gewidmet Theodor Billroth (Contributions to the surgery commemorative publication dedicated to Theodor Billroth) 610-630, 1892 (in German).

3. Kawaguchi K, Koike M, Tsuruta K, Okamoto A, Tabata I, Fujita N. Lymphoplasmacytic sclerosing pancreatitis with cholangitis: a variant of primary sclerosing cholangitis extensively involving pancreas. Hum Pathol 22: 387-395, 1991.

4. Hamano H, Kawa S, Horiuchi A, et al. High serum IgG4 concentrations in patients with sclerosing pancreatitis. N Engl J Med 344: 732-738, 2001.

5. AbdelRazek MA, Venna N, Stone JH. IgG4-related disease of the 
central and peripheral nervous systems. Lancet Neurol 17: 183192, 2018.

6. Ohyama K, Koike H, Iijima M, et al. IgG4-related neuropathy: a case report. JAMA Neurol 70: 502-505, 2013.

7. Nehring P, Przybyłkowski A. Think twice before operating on a pancreatic mass: could it be $\operatorname{IgG} 4-$ related disease? Lancet 395: $816,2020$.

8. Goto H, Takahira M, Azumi A; Japanese Study Group for IgG4Related Ophthalmic Disease. Diagnostic criteria for IgG4-related ophthalmic disease. Jpn J Ophthalmol 59: 1-7, 2015.

9. McNab AA. Orbital myositis: a comprehensive review and reclassification. Ophthalmic Plast Reconstr Surg 36: 109-117, 2020.

10. Baptista B, Casian A, Gunawardena H, D'Cruz D, Rice CM. Neurological manifestations of IgG4-related disease. Curr Treat Options Neurol 19: 14, 2017.

11. Inoue $\mathrm{D}$, Zen $\mathrm{Y}$, Sato $\mathrm{Y}$, et al. IgG4-related perineural disease. Int J Rheumatol 2012: 401890, 2012.

12. Collins MP, Dyck PJB, Gronseth GS, et al. Peripheral Nerve Society Guideline on the classification, diagnosis, investigation, and immunosuppressive therapy of non-systemic vasculitic neuropathy: executive summary. J Peripher Nerv Syst 15: 176-184, 2010

13. Ohyama K, Koike H, Takahashi M, et al. Immunoglobulin G4related pathologic features in inflammatory neuropathies. Neurology 85: 1400-1407, 2015.

14. Yokoi S, Kawagashira Y, Ohyama K, et al. Mononeuritis multiplex with tumefactive cellular infiltration in a patient with reactive lymphoid hyperplasia with increased immunoglobulin G4-positive cells. Hum Pathol 45: 427-430, 2014.

15. Suzuki Y, Shiraishi M, Yamada K, Doi M, Kato M, Hasegawa Y. A case of refractory IgG4-related peripheral neuropathy with severe axonal damage. Clin Neurol 56: 323-327, 2016.

16. Khosroshahi A, Wallace ZS, Crowe JL, et al. Second International Symposium on IgG4-Related Disease. International Consensus Guidance Statement on the Management and Treatment of IgG4Related Disease. Arthritis Rheumatol 67: 1688-1699, 2015.

The Internal Medicine is an Open Access journal distributed under the Creative Commons Attribution-NonCommercial-NoDerivatives 4.0 International License. To view the details of this license, please visit (https://creativecommons.org/licenses/ by-nc-nd/4.0/).

(C) 2021 The Japanese Society of Internal Medicine

Intern Med 60: 1941-1947, 2021 